INRA Prod. Anim.

1996, 9 (2), 103-111

\section{VEISSIER}

INRA Laboratoire Adaptation

des Herbivores aux Milieux

Theix 63122 Saint-Genès Champanelle

\title{
Intérêts de l'analyse comportementale dans les études de bien-être : le cas des veaux de boucherie
}

\author{
Le bien-être des animaux d'élevage est devenu une préoccupation \\ importante dans nos sociétés. Les modes de production d'œufs, de \\ viande de porc et de veau sont particulièrement critiqués. Le rôle du \\ scientifique est de proposer des mesures objectives du bien-être et de \\ l'adaptation des animaux à leurs conditions d'élevage. Les mesures \\ comportementales font partie de l'arsenal des critères utilisables. Cet \\ article en présente les avantages et les limites d'utilisation en \\ s'appuyant sur des exemples pris chez les veaux.
}

Depuis quelques décennies, le confort des animaux d'élevage est progressivement devenu une préoccupation importante du grand public. Dans le même temps, certains élevages ont été profondément modifiés. Ainsi, avec la spécialisation des élevages bovins laitiers, la production de viande de

\section{Résumé}

Nous partons du principe que le bien-être d'un individu est satisfait s'il peut s'adapter à son environnement. Cette condition est respectée quand l'individu peut satisfaire ses besoins sans souffrir ou s'épuiser. Dans ce domaine, où il est indispensable d'éviter l'anthropomorphisme, le rôle du scientifique est d'évaluer les besoins de l'animal et de proposer des mesures objectives de son adaptation. Ces mesures peuvent être d'ordre sanitaire, zootechnique, neuro-endocrinien et comportemental. Les critères comportementaux présentent l'avantage d'être très sensibles et spécifiques et de varier avec l'intensité de la contrainte imposée à l'animal.

Ils permettent de prévenir l'apparition de troubles cliniques (les rythmes d'activité sont modifiés avant l'état sanitaire ou la production) et de déceler de faibles variations (la réactivité peut augmenter lorsque l'environnement est pauvre sans que les critères physiologiques varient). Les indices comportementaux sont spécifiques : ils renseignent sur la nature de la contrainte. Ainsi les postures de repos renseignent sur la place allouée à l'animal, et les activités orales non nutritives sur le manque d'activité. Enfin, dans certains cas, la fréquence d'un comportement est liée à l'intensité de la contrainte. Cependant, l'interprétation d'un ensemble d'indicateurs - comportementaux et autres - permet seule de rendre compte des sollicitations de l'organisme.

Ces aspects sont illustrés à partir du modèle du veau chez qui les contraintes les plus marquantes sont : le manque de place, l'alimentation uniquement lactée et la restriction des contacts sociaux. veau est devenue plus importante en quantité et s'est transformée. Alors qu'auparavant la viande de veau provenait essentiellement d'animaux de race bouchère ou mixte nourris au lait de leur mère, elle provient à l'heure actuelle essentiellement d'animaux de race laitière spécialisée nourris avec un aliment liquide " reconstitué ». Autrefois engraissés sur leur lieu de naissance, les veaux destinés à la boucherie sont maintenant élevés dans des ateliers spécialisés. La concomitance de la demande sociale de protection des animaux d'élevage et de l'évolution des conditions de production des veaux de boucherie explique que cet élevage soit particulièrement critiqué. Ce débat de société implique à la fois le consommateur, le producteur, le grand public et le législateur. Le rôle du scientifique est de fournir des éléments objectifs qui devraient servir de base de discussion entre ces différents intervenants.

Nous partons du principe que le bien-être d'un individu est respecté s'il peut s'adapter à son environnement (voir les définitions du bien-être dans l'encadré). Cette condition est respectée quand l'environnement satisfait d'emblée tous les besoins de l'individu. Cet environnement idéal ne peut être que théorique car on ne connaît pas l'ensemble des besoins des animaux ; de plus, ces besoins varient d'un individu à un autre et certains besoins peuvent ne pas être compatibles avec d'autres. Le bien-être est également respecté 


\section{Le bien-être animal}

Nous avons choisi trois définitions :

Dawkins (1983a) : le bien-être est l'absence de souffrance, la souffrance incluant des états émotionnels désagréables tels la peur, l'ennui, la douleur et la faim. Cet auteur souligne l'aspect subjectif du bien-être (tout dépend de la perception que l'individu a de son environnement) mais propose des moyens objectifs de mesure de cette subjectivité (utilisation des conditionnements par exemple).

Broom (1986) : le bien-être d'un individu est défini en regard des efforts qu'il doit fournir pour s'adapter à l'environnement. Si l'adaptation demande peu d'efforts, le bien-être est satisfait ; si l'individu s'épuise en s'adaptant ou qu'il ne parvient pas à s'adapter, le bien-être n'est pas satisfait. Broom suggère donc qu'il y a un continuum entre un environnement idéal et un environnement inacceptable.

Jensen (1995) souligne les difficultés de rendre opérationnelle la définition de Broom car nous ne disposons pas de moyens d'évaluation des efforts d'adaptation. Selon lui, l'inadaptation provient d'un rétro-contrôle par les actions de l'animal qui n'est plus suffisant pour éliminer les motivations qui sont à l'origine de ces actions. Le bien-être serait donc respecté tant que l'individu peut trouver une réponse comportementale efficace.

si l'environnement n'est pas idéal mais que l'individu peut satisfaire ses besoins sans s'épuiser ou souffrir. La notion de souffrance peut elle-même paraître subjective. Selon Dawkins (1983a), une inadaptation totale aboutit à des troubles cliniques (atteinte de la croissance, pathologies), ce qui ne signifie pas pour autant qu'un individu en bonne santé ne souffre pas : cet individu peut présenter certaines anomalies physiologiques ou comportementales qui suggèrent que, si la situation se prolongeait, des troubles cliniques apparaîtraient. La souffrance peut donc être interprétée comme un moyen de prévenir l'animal des troubles cliniques.

Les recherches dans le domaine du bienêtre animal doivent avant tout éviter l'anthropomorphisme. Ainsi, ce n'est pas parce qu'une situation semble agréable à un humain qu'elle est jugée comme telle par l'animal. Un exemple frappant concerne les préférences de sol chez les poules. Bien que le grillage apparaisse à nos yeux moins confortable qu'un sol percé de trous (préconisé en Grande-Bretagne dans le rapport Brambell 1965), les poules préfèrent le grillage (Hughes et Black 1973). L'objet des recherches est d'évaluer les besoins de l'animal et de déterminer des mesures objectives de son adaptation, de manière à pouvoir déceler un dépassement de ses capacités. Ces mesures reposent sur des critères : sanitaires (apparition de pathologies), zootechniques (baisse du niveau de production), physiologiques (activation des réponses neuro-endocriniennes de stress) et comportementaux (pour revues, voir : Dantzer et Mormède 1979, Stephens 1980, Dawkins 1983a, Broom et Johnson 1993). Cet article s'intéressera essentiellement aux indicateurs comportementaux qui, outre leur facilité de mise en œuvre, présentent l'avantage d'être très sensibles, spécifiques et de varier avec l'intensité de la contrainte imposée à l'animal. Cette courte synthèse ne se veut pas une revue exhaustive de toutes les mesures comportementales utilisables pour la question du bien-être animal. Son but est uniquement d'illustrer l'intérêt des mesures comportementales à l'aide du modèle du veau de boucherie, qui est soumis à des contraintes spatiales, alimentaires et sociales. Des conclusions équivalentes pourraient bien sûr être tirées de résultats obtenus chez d'autres espèces.

\section{1 / Sensibilité}

Lorsque les animaux sont soumis à une contrainte légère, les comportements peuvent être modifiés sans que les paramètres physiologiques, zootechniques ou sanitaires soient affectés. Les observations comportementales peuvent donc servir pour déceler de faibles différences entre des situations.

Ainsi, dans une étude visant à déterminer l'impact de la pauvreté de l'environnement dans les cases individuelles, nous avons comparé des veaux pouvant avoir plus ou moins de contacts avec leurs voisins et ayant ou non à leur disposition des objets faciles à grignoter (pneu, chaîne). Les parois entre cases étaient soit à claire-voie (permettant aux voisins de se voir, flairer, toucher et lécher), soit pleines (permettant les contacts uniquement par l'avant de la case), soit pleines et prolongées à l'avant des cases (empêchant tout contact). A la fois des indices sanitaires (nombres de jours de traitements médicaux), zootechniques (croissance), physiologiques (réponse de l'axe corticotrope à une stimulation par de l'ACTH exogène, enzymes de synthèse des catécholamines) et comportementales (emploi du temps, réactivité) ont été utilisés. L'emploi du temps était calculé à partir d'observations par pointage toutes les 10 minutes. Les tests de réactivité ont été inspirés des travaux de Broom (1987) chez la truie (test de la douche). Un jet d'eau produit par un lave-glace était envoyé pendant une seconde sur le dos d'un animal couché ; le jet était commandé à distance, de sorte que l'observateur n'était pas visible des animaux. La réaction était appréciée sur une échelle allant de 0 : pas de réaction, à 4 : l'animal se lève. Les résultats montrent que la restriction des contacts sociaux augmente la réactivité ; l'absence d'objet augmente la fréquence des grignotages du cornadis, des léchages de babines et des jeux de langue : les veaux chercheraient à compenser les grignotages du pneu et de la chaîne par d'autres activités 
Tableau 1. Influence de la richesse de l'environnement sur des indicateurs sanitaires, zootechniques, physiologiques et comportementaux d'inconfort chez des veaux en cases individuelles. Les comparaisons portent sur des veaux disposant de jouets à mordiller (pneu, chaîne) et séparés les uns des autres soit par des parois à claire-voie (permettant les contacts entre voisins), soit par des parois pleines (seuls des contacts à l'avant de la case sont possibles), soit par des parois pleines et prolongées à l'avant des cases (aucun contact entre veaux) et des veaux privés à la fois de contacts et de jouets.

\begin{tabular}{|c|c|c|c|c|c|}
\hline \multirow{2}{*}{$\begin{array}{c}\text { Type de parois entre cases } \\
\text { Présence de jouets }\end{array}$} & \multirow{2}{*}{$\begin{array}{c}\text { A claire-voie } \\
\text { oui } \\
n=16\end{array}$} & \multirow{2}{*}{$\begin{array}{c}\text { Pleines } \\
\text { oui } \\
\mathrm{n}=16\end{array}$} & \multicolumn{2}{|c|}{ Pleines et prolongées } & \multirow{2}{*}{$\begin{array}{l}\text { Ecart } \\
\text { type }\end{array}$} \\
\hline & & & $\begin{array}{c}\text { oui } \\
\mathrm{n}=16\end{array}$ & $\begin{array}{c}\text { non } \\
\mathrm{n}=16\end{array}$ & \\
\hline GMQ (kg) & 1,29 & 1,32 & 1,28 & 1,27 & 0,12 \\
\hline $\mathrm{Nb}$ de jours de traitement & 5,56 & 2,88 & 2,56 & 3,06 & 3,47 \\
\hline Cortisol 30 min après ACTH $(\mathrm{ng} / \mathrm{ml})$ & 16,48 & 18,14 & 19,99 & 20,65 & 8,47 \\
\hline TH (ng/h/mg protéine) $)^{(1)}$ & 3,24 & 3,53 & 4,83 & 4,01 & 1,61 \\
\hline PNMT (ng/h/mg protéine) $)^{(1)}$ & 0,23 & 0,25 & 0,23 & 0,26 & 0,14 \\
\hline Grignotages jouets (\% temps) & 7,1 & 9,8 & 5,2 & & \\
\hline Grignotages cornadis (\% temps) & $2,2 \mathrm{a}$ & $3,0 \mathrm{a}$ & $3,4 \mathrm{a}$ & $5,1 \mathrm{~b}$ & 2,2 \\
\hline Léchage babines (\% temps) & $2,7 \mathrm{a}$ & $3,1 \mathrm{a}$ & $3,7 \mathrm{a}$ & $5,2 \mathrm{~b}$ & 2,1 \\
\hline Jeux de langue (\% temps) & $0,3 \mathrm{a}$ & $0,8 \mathrm{a}$ & $0,6 \mathrm{a}$ & $2,1 \mathrm{~b}$ & 0,9 \\
\hline Réactivité(2) $^{2}$ & $2 \square \mathrm{a}$ & $3 a b$ & $8 \mathrm{~b}$ & $8 \mathrm{~b}$ & \\
\hline
\end{tabular}

(1) Pour ces mesures, $\mathrm{n}=8$ dans chaque lot, TH : tyrosine hydroxylase et PNMT : Phenyl-N-Méthanolamine-Transférase, $\mathrm{ng} / \mathrm{h} / \mathrm{mg}$ protéine : nanogrammes de substrat par heure et par milligramme de protéines contenues dans l'échantillon.

(2) Nombre d'animaux présentant des réactions intenses lorsqu'ils reçoivent un jet d'eau.

orales non nutritives (tableau 1). Aucun indice autre que comportemental n'a varié significativement entre les traitements. Pour cet essai, notre conclusion a été que les contacts sociaux et la présence d'objets manipulables pourraient améliorer les conditions de vie des veaux, mais de façon relativement modérée. L’idée que certains indices comportementaux sont plus sensibles que les mesures neuro-endocriniennes classiques de stress est confirmée par les résultats de Friend et al (1985) et Dellmeier et al (1985) : les niveaux d'hormone thyroïdienne ou de corticoïdes ne permettent pas de distinguer des veaux en huttes individuelles de veaux en groupes ; par contre, lorsqu'ils en ont la possibilité, les veaux en hutte se déplacent plus et ont plus d'interactions sociales, signe d'une plus grande motivation pour ces activités. De la même façon, de Passillé et Rushen (1995) ont montré que la restriction spatiale modifie la motivation pour les déplacements mais pas l'activité de l'axe corticotrope (responsable de la production de cortisol). Dans d'autres cas où les différences entre traitements sont plus importantes - par exemple, veaux à l'attache, sur caillebotis et avec une surface par animal réduite comparés à des veaux en groupes, dans des cases paillées avec $1,5 \mathrm{~m}^{2}$ par animal, on note au contraire des variations significatives des indicateurs physiologiques (Dantzer et al 1983).

La sensibilité des indicateurs comportementaux permet de les utiliser comme des indicateurs précoces. Ainsi, nous avons observé l'emploi du temps de génisses venant d'être sevrées et rentrées à l'étable (Veissier et al 1989). Par le jeu d'une analyse factorielle des correspondances, nous avons calculé un indice représentant le niveau d'activité. Dès la rentrée à l'étable, des pics d'activité sont visibles au moment de la distribution des repas et l'activité est plus faible la nuit qu'à tout autre moment (figure 1). La différence de niveau d'activité entre les pics et la nuit ou la journée est faible le jour même de la rentrée à l'étable $(\mathrm{D}=2,3$ pour la différence matin - journée) elle s'accentue progressivement après la rentrée à l'étable ( $\mathrm{D}=6,8$ au quatrième jour dans l'étable), ce

\section{La restriction des contacts avec les congénères et l'absence d'objet modifie les critères comportementaux sans qu'il y ait d'effet sur les paramètres physiologiques.}

Figure 1. Variations circadiennes d'activité chez des génisses. Le niveau d'activité est calculé à partir d'observations par pointages à l'aide de l'équation : - 0,67\% temps passé " couché tête posée » - 0,46\% temps passé " couché tête levée » + 0,41\% temps passé « debout immobile » + 0,53\% temps passé " debout actif sans manger » $+0,89 \%$ temps passé en mangeant.

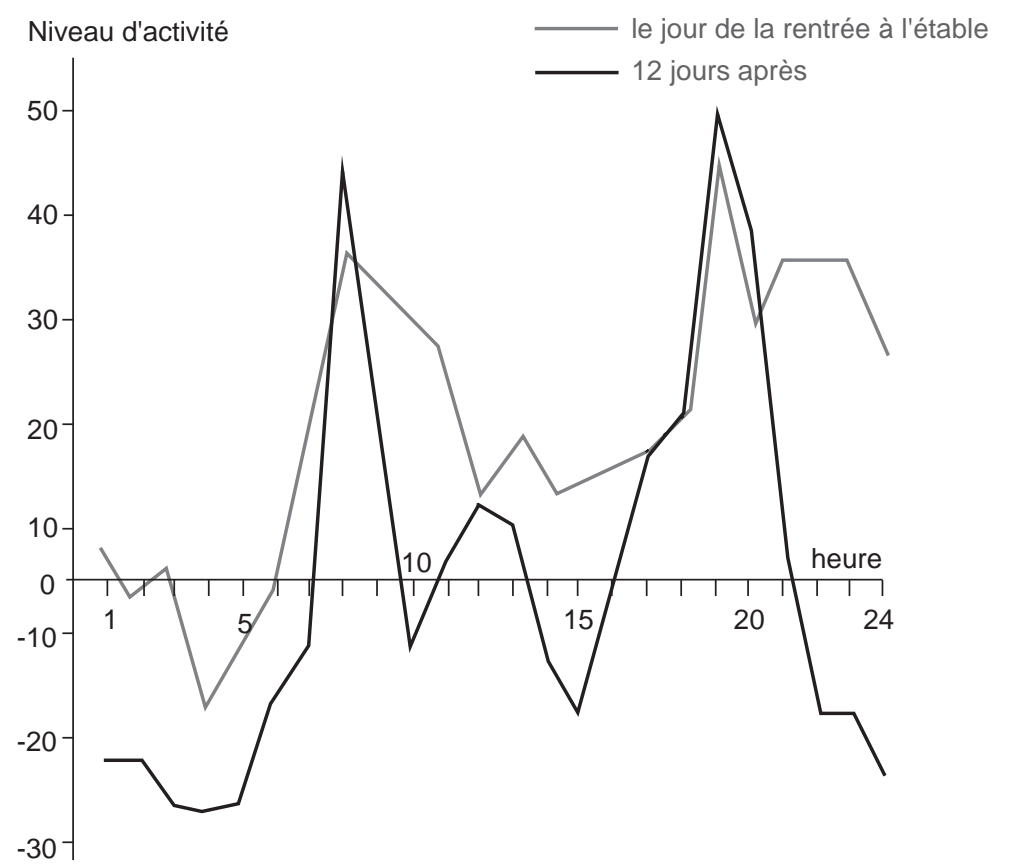


qui était la tendance attendue. Mais, un résultat plus intéressant a été obtenu du fait d'un épisode de troubles respiratoires. Les symptômes cliniques (toux, fièvre) ont été observés au huitième jour après l'arrivée des animaux. Or, au septième jour, la quantité de fourrage ingérée par les génisses était diminuée de $24 \%$ par rapport aux jours précédents. Dès le sixième jour, la différence d'activité entre les pics et la nuit avait diminué $(\mathrm{D}=5,0$ puis 4,1 au sixième et neuvième jours $)$ ; les valeurs sont redevenues normales par la suite. Cette altération du rythme était plus marquée chez les génisses Aubrac et Salers que chez les Pie-noir, lesquelles ont présenté des symptômes d'atteinte respiratoire moins intenses.

La grande sensibilité des indicateurs comportementaux leur confère donc une valeur de prédiction de troubles cliniques.

\section{2 / Spécificité}

Lorsqu'elles ont été mises en évidence, les réactions neuro-endocriniennes face à une agression ont été considérées comme non spécifiques (travaux de Cannon et de Selye, rapportés par Dantzer et Mormède 1979). Par la suite, la non-spécificité de ces réponses a été fortement discutée (pour revue : Dantzer et Mormède 1983). On distingue les individus selon des profils réactionnels : certains répondent plutôt par une activation du système sympathique associée à des réponses de fuite ou d'agression ("active copers ») alors que d'autres répondent au contraire par une activation de l'axe corticotrope et une inhibition comportementale (" passive copers ») (Henry et Stephens 1977, cités par Dantzer et Mormède 1979, Korte et al 1992). Certaines situations favorisent les réponses actives et d'autres au contraire, les réponses passives, comme les épreuves de conditionnement d'évitement actif ou passif (pour exemples chez les porcs : Mormède et Dantzer 1977, Dantzer et Mormède 1978). Toutefois, dans l'état actuel des connaissances, les indicateurs physiologiques utilisés dans les études de bien-être renseignent peu sur la nature des contraintes imposées aux animaux. Il est probable que ce manque de spécificité est dû au fait que les scientifiques qui s'intéressent au bien-être animal recherchent souvent l'indicateur physiologique universel. Ceci expliquerait l'utilisation très fréquente du taux de cortisol plasmatique. Or celui-ci reflète plutôt une activation de l'organisme, que cette activation soit liée à un contexte aversif ou positif. Ainsi, le cortisol augmente à la fois lorsque l'animal reçoit des chocs électriques (Haltmeyer et al 1967, Kant et al 1985) et lorsqu'il est engagé dans un comportement sexuel (Gonzales 1989).

A l'inverse, les indicateurs comportementaux renseignent généralement sur la nature de la contrainte imposée aux animaux. Ainsi, la restriction de la place se traduit par des modifications posturales : en cases étroites, les veaux se déplacent moins et se couchent avec les membres plus souvent pliés que dépliés (de Wilt 1985, Webster et al 1985, Le Neindre 1993, figure 2). Selon les travaux de Ketelaar-de Lauwere et Smits (1991), un veau de plus de 18 semaines ne peut pas étendre une patte arrière loin du corps si sa case ne mesure pas au moins $90 \mathrm{~cm}$ de large (figure 3).

Par contre, il est illusoire de vouloir utiliser ces critères pour étudier l'effet d'autres contraintes comme le mode d'alimentation. Aucune modification posturale n'est observée entre des veaux nourris au seau et des veaux nourris avec un distributeur automatique muni de tétines (Bertrand et Martineau 1993). Pour cette comparaison, il est plus judicieux de s'intéresser aux activités orales

Figure 2. Postures de repos chez les veaux (d'après de Wilt 1985).

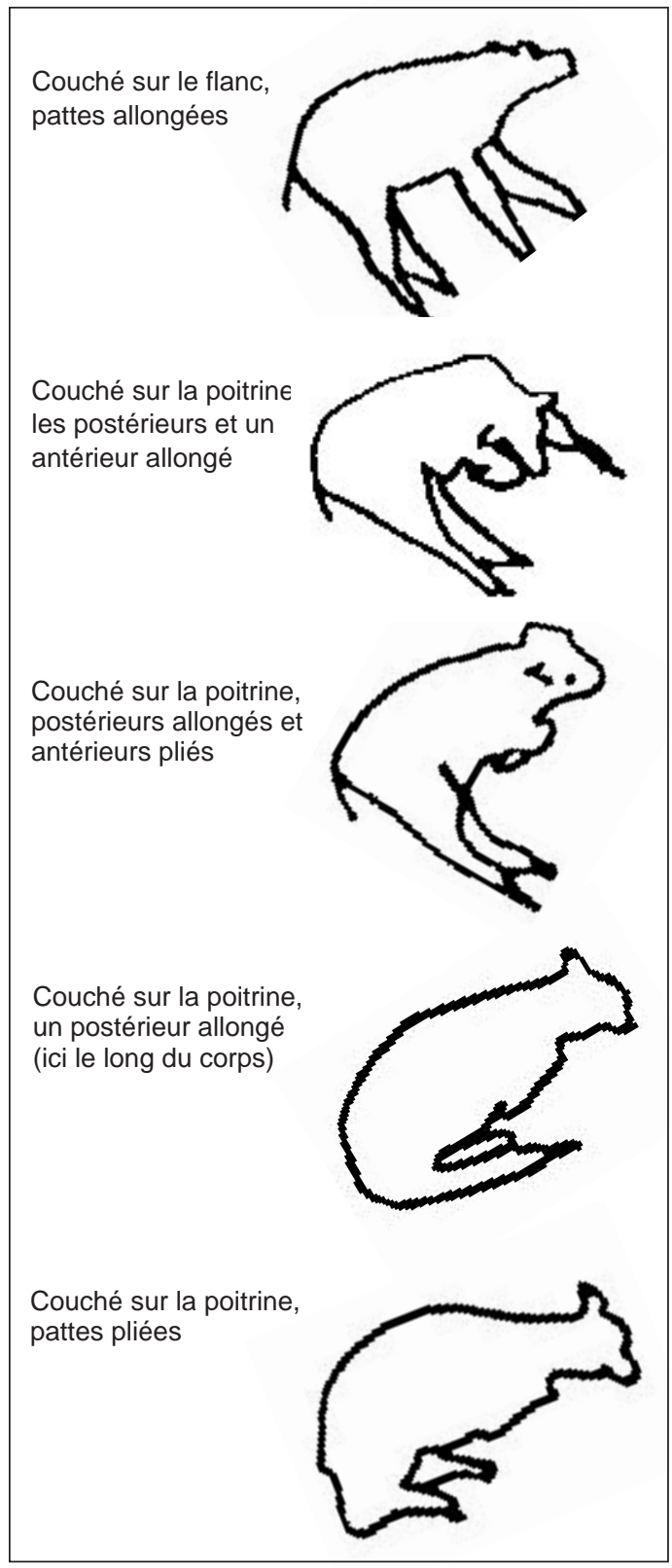


Figure 3. Variations du temps passé couché avec un membre postérieur déplié loin du corps en fonction de la dimension des cases individuelles (d'après Ketelaar-de Lauwere et Smits 1991).

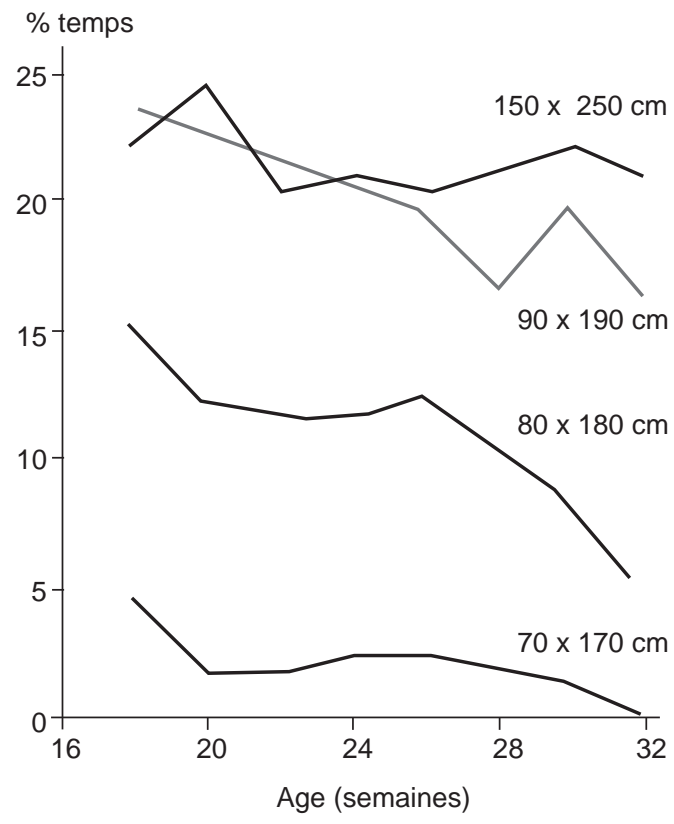

(Kopp et al 1986). En effet, selon Hammel et al (1988), il existe vraisemblablement une motivation importante pour la succion et si cette motivation n'est pas assouvie lors du repas, les veaux compensent par des succions non-nutritives (tableau 2). De la même façon, la fréquence des jeux de langue et celle des manipulations d'objets varient avec le mode d'alimentation (Kooijman et al 1991).

Tableau 2. Temps passé à ingérer le lait et à sucer une tétine non nutritive chez des veaux recevant du lait ad libitum soit au seau soit au moyen d'une tétine reliée à un container (d'après Hammel et al 1988).

\begin{tabular}{|l|c|c|}
\hline \multirow{2}{*}{ Temps passé (min) } & \multicolumn{2}{|c|}{ Veaux nouris } \\
\cline { 2 - 3 } & $\begin{array}{c}\text { au seau } \\
(\mathrm{n}=8)\end{array}$ & $\begin{array}{c}\text { à la tétine } \\
(\mathrm{n}=6)\end{array}$ \\
\hline Ingestion du lait & $17,7 \pm 11,7 \mathrm{a}$ & $44,2 \pm 11,8 \mathrm{~b}$ \\
Succion d'une tétine & & $0,4 \pm 0,3 \mathrm{~b}$ \\
- pendant les repas & $8,3 \pm 12,0 \mathrm{a}$ & $0,6 \pm 0,2 \mathrm{~b}$ \\
- en dehors des repas & $4,6 \pm 1,3 \mathrm{a}$ & $0,6 \pm 0$ \\
\hline
\end{tabular}

L'expérimentateur devra donc tenter de comprendre comment une situation est perçue par l'animal pour orienter le choix des mesures comportementales à entreprendre.

\section{3 / Proportionnalité}

La plupart des indices comportementaux varient de façon proportionnelle avec l'intensité de la contrainte. Pour mettre en évidence
Figure 4. Utilisation de la place en fonction de la surface théorique disponible (taille de la case / nombre de veaux dans la case) pour des veaux sur caillebotis. L'indice de déploiement des pattes est calculé à partir d'observations par pointages à l'aide de l'équation : 0,79 temps passé couché avec tous les membres dépliés + 0,66 temps passé debout en déplacement $+0,32$ temps passé couché avec les deux postérieurs dépliés + 0,17 temps passé couché avec deux ou trois membres dépliés $+0,08$ temps passé couché avec un postérieur déplié + 0.01 temps passé debout immobile - 0,04 temps passé couché avec un ou les deux antérieurs dépliés - 0,07 temps passé couché avec tous les membres pliés.

Indice de déploiement

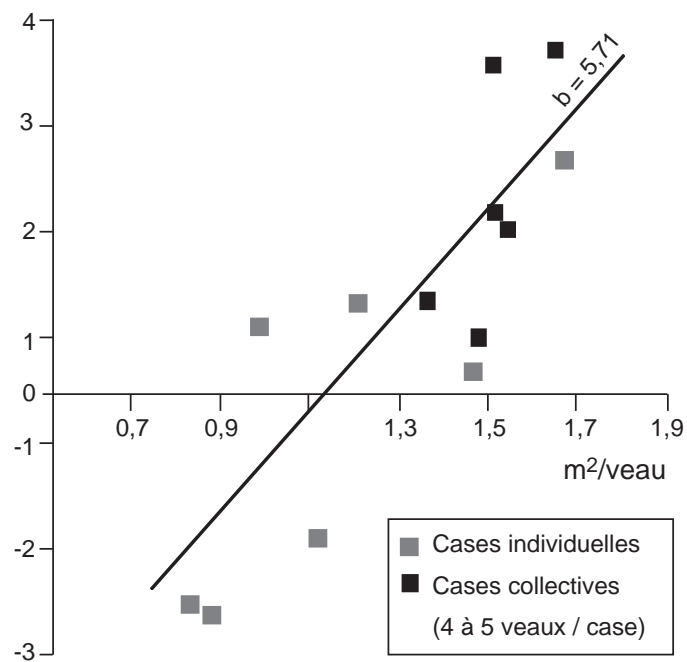

cette proportionnalité, il est parfois nécessaire de recourir à des indices synthétiques. En effet, lorsqu'une contrainte s'intensifie, la nature des réponses comportementales peut évoluer. Pour reprendre l'exemple trivial de l'étroitesse des cases, on imagine aisément que lorsqu'il ne dispose pas d'assez de place pour étendre ses membres, un veau puisse commencer par plier un, deux, voire trois membres avant de les plier tous. Les données reportées par Le Neindre (1993) confirment cette hypothèse : à l'âge de 13 semaines, un veau élevé dans une case de $55 \mathrm{~cm}$ de large se couche avec un antérieur plié moins souvent qu'un veau élevé dans une case de $65 \mathrm{~cm}$ de large (21,0 contre $30,5 \%$ du temps) mais il se couche plus souvent avec tous les membres pliés (41,0 contre $33,2 \%$ du temps). Une analyse factorielle des correspondances de données portant sur des veaux entretenus dans des cases de taille variable nous a permis de définir un indice synthétique de déploiement des pattes, calculé à partir du temps passé dans les différentes postures. On obtient une relation positive entre cet indice et la surface théorique mise à disposition du veau (figure 4). Cette relation peut être considérée comme linéaire pour des surfaces comprises entre 0,9 et $1,6 \mathrm{~m}^{2} / \mathrm{veau}$. Toutefois, l'indice de déploiement des pattes ne peut varier

\section{La plupart des indices comportementaux varie proportionnellement à l'intensité de la contrainte.}


qu'entre deux extrêmes : la borne inférieure correspond au point où le veau serait toujours debout immobile ou couché avec les quatre membres pliés, et la borne supérieure, au point où le veau serait toujours en déplacement ou couché avec tous les membres dépliés. Cet extrême n'est jamais atteint car un animal diversifie toujours ses postures. Néanmoins, il est fort probable qu'au-delà d'une certaine surface, le coefficient de déploiement des pattes n'évolue plus, l'animal ayant alors suffisamment de place pour adopter les postures qui lui plaisent. D'après nos observations, les limites inférieure et supérieure de ce coefficient avoisineraient -3 et 10 respectivement, pour des veaux sur caillebotis. La définition de la plus petite taille de case permettant d'atteindre la limite supérieure du coefficient de déploiement des pattes devrait aider au choix d'une taille minimale pour une case à veau. Par ailleurs, non seulement la taille mais également la forme des cases ainsi que le nombre de veaux par case ou le type de sol est susceptible d'influencer la place que le veau pourra effectivement utiliser. L'analyse des variations du coefficient de déploiement des pattes pourra donc permettre d'optimiser la surface d'un bâtiment.

\section{4 / Subjectivité ?}

On reproche parfois aux mesures comportementales un manque d'objectivité. Une action est parfois considérée comme la traduction d'un inconfort parce qu'elle apparaît anormale à nos yeux. Ainsi, certains auteurs ont qualifié les jeux de langue de stéréotypies. Une stéréotypie est une action fixe, répétée et sans but apparent (Odberg 1987) sous la

Figure 5. Variations des léchages/grignotages d'objets et des jeux de langue en fonction de l'alimentation chez des veaux de 3 mois (d'après Kooijman et al 1991).

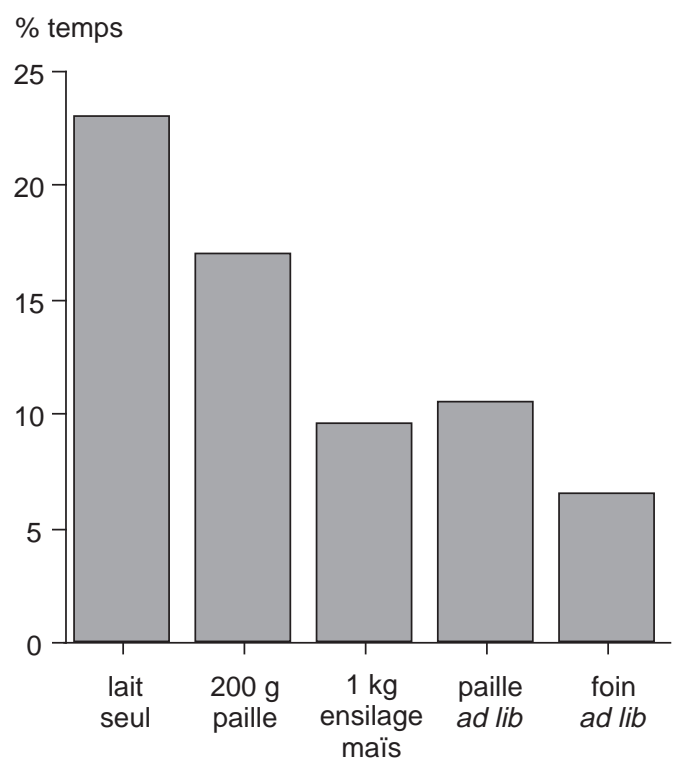

dépendance des systèmes opioïdes et dopaminergiques (Sahakian et al 1975, Kennes et al 1988, Savory et al 1992). Or, les jeux de langue ne semblent pas être des actions aussi répétitives que les stéréotypies observées dans d'autres espèces (mordillement de barres chez les truies à l'attache : Cronin et al 1985, Terlouw et al 1991, va-et-vient chez les visons : Mason 1993, sauts dans les cages chez les surmulots : Odberg 1987) et, à notre connaissance, aucune étude n'a été entreprise sur leur mécanisme d'apparition. Par ailleurs, selon un raisonnement circulaire, certaines actions sont considérées comme indicatrices d'inconfort parce que leur fréquence est augmentée dans des situations que nous jugeons a priori inconfortables. Des contradictions peuvent alors émerger. Selon les résultats précédemment cités de Kooijman et al (1991), les léchages ou grignotages d'objets révèlent un inconfort puisqu'ils diminuent lorsque l'alimentation est diversifiée (figure 5). Or la conclusion inverse émerge des données de Le Neindre (1993) puisque la fréquence de ces activités est augmentée lorsque les veaux disposent de plus de place (1,2 contre $0,3 \%$ du temps chez des veaux en cases de 110 et $55 \mathrm{~cm}$ de large respectivement). Plus qu'une contradiction, ces interprétations divergentes soulignent la nécessité de bien connaître l'origine d'un comportement avant de l'utiliser dans les études de bienêtre.

\section{Conclusions - Perspectives}

De par leur sensibilité et leur spécificité, les indices comportementaux sont donc une aide précieuse pour comprendre comment un individu s'adapte à son environnement. Toutefois, dans le cadre des études relatives au bien-être animal, ces indices doivent être utilisés avec précaution compte tenu de l'impérative nécessité d'éviter tout anthropomorphisme ou toute conclusion hâtive du fait d'une mauvaise compréhension de l'origine de modifications observées. De plus, la grande sensibilité des mesures comportementales nécessite d'effectuer les observations sur de nombreux animaux et sur une longue période (Dawkins 1983a, Eddison 1995).

Le fait que les indicateurs physiologiques soient parfois décevants ne doit pas entraîner leur abandon dans les études visant à cerner l'impact de conditions d'élevage. Cela signifie plutôt qu'il est nécessaire d'approfondir les données scientifiques dans ce domaine (Dantzer et Mormède 1983, Rushen 1991). En particulier, la physiologie du stress chronique chez les animaux d'élevage reste mal connue. Par ailleurs, des recherches de physiopathologie pourraient aider à interpréter certaines réponses comportementales, comme les activités orales non-nutritives actuellement considérées comme anormales chez les veaux.

Bien que les indices comportementaux soient une aide précieuse pour la question du 
bien-être animal, le choix d'un mode d'élevage doit reposer sur un ensemble de critères. En effet, production, comportement, pathologie et physiologie ne vont pas toujours dans le même sens. Ainsi, l'élevage des veaux en groupes peut sembler plus confortable puisqu'il permet aux animaux d'interagir socialement. Toutefois, la fréquence des affections respiratoires et digestives est accrue par rapport aux veaux en cases individuelles (deWilt 1985, Smits et Ham 1988, Webster 1991, van de Braak et Kleinhout 1991). Cette ambiguïté a suscité un ensemble de travaux visant à étudier les différentes contraintes imposées aux veaux dans les cases individuelles : contrainte de place (dePassillé et Rushen 1995) et contrainte sociale (Veissier et al 1994 $a$ et $b$ ). Ces études suggèrent que les différences comportementales et physiologiques relevées entre cases collectives et cases individuelles proviennent en majeure partie de surfaces par animal différentes entre les deux systèmes (elle est généralement plus élevée dans les cases collectives) et que des améliorations de la case individuelle sont possibles si on permet les contacts entre veaux.

Dans cet article, nous nous sommes attachés à présenter les façons dont les animaux pouvaient s'adapter à des contraintes environnementales. Définir le bien-être comme une absence de souffrance est certainement réducteur, pour pouvoir affirmer avec certitude que l'animal " apprécie » la situation dans laquelle il se trouve, il nous faudrait disposer de mesures d'indicateurs de "satisfaction "de l'animal et ce, à la fois dans le court et le long terme. Or, il n'existe pas de mesures de bien-être à proprement parler. Même si on attribue aux endorphines une valeur hédonique, leur signification biologique reste encore incertaine. Une autre façon d'aborder la question du bien-être animal serait d'étudier les préférences des animaux, ce qui est fait dans des épreuves de choix ou de conditionnement (Matthews et Temple 1979, Dawkins 1983b, Faure 1986). Là encore, cette voie de recherche présente des difficultés. Ainsi, il est nécessaire de s'assurer qu'un résultat négatif ne provient pas d'une absence de compréhension du test par l'animal (Faure et Mills 1995). De plus, les préférences des animaux varient en fonction de leur expérience passée : l'individu choisit bien souvent l'environnement auquel il est habitué (pour discussion : voir Dawkins 1983a).

Les travaux concernant le bien-être devraient aider les prises de décision en matière de protection animale. Dans certains cas, lorsque l'on diminue progressivement la contrainte, la réponse de l'animal ne varie plus au-delà d'un seuil, comme dans l'exemple des postures des veaux que nous avons cité à plusieurs reprises. Il est possible de proposer ce seuil comme une limite endeçà de laquelle la situation ne peut pas être jugée optimale. Aussi n'est-il pas surprenant que la réglementation communautaire ait imposé $90 \mathrm{~cm}$ comme largeur minimale des cases à veaux, cette taille étant en accord avec les données scientifiques (travaux de Ketelaar-de Lauwere et Smits 1991, cités précédemment). Dans d'autres cas au contraire, il n'est pas possible de mettre en évidence un optimum. Le scientifique peut tout au plus classer les situations qu'il étudie sur une échelle ordinale. A partir de ce classement, la définition du niveau acceptable revient à la société car il s'agit d'une décision subjective qui ne peut donc être prise par le scientifique (Rutter 1995). Enfin, le classement ordinal peut être impossible, soit que les critères d'appréciation de l'inconfort sont divergents (voir l'exemple des veaux en cases collectives), soit que les modalités de réponse varient d'une situation à une autre (voir la divergence d'interprétation des activités orales). Les résultats biologiques ne peuvent alors être utilisés pour répondre à la demande de protection des animaux qu'en référence à la norme sociale qui la produit. C'est pourquoi une réflexion sur les déterminants sociologiques de la demande actuelle de respect du bien-être animal doit être entreprise.

\section{Remerciements}

Je remercie P. Mormède pour sa lecture critique du manuscrit.

\section{Références bibliographiques}

Bertrand G., Martineau C., 1993. Intérêt de l'élevage du veau de boucherie alimenté par un distributeur automatique. Institut de l'Elevage, 45 pp., Paris.

Brambell report, command paper, 1965. Report of the technical committee to enquire into the welfare of animals kept under intensive livestock husbandry systems. H.M.S.O., London.

Broom D.M., 1986. Indicators of poor welfare. Br. Vet. J., 142, 524-526.

Broom D.M., 1987. Applications of neurobiological studies to farm animal welfare. In : P.R. Wiepkema and P.W.M. Van Adrichen (Editors), Biology of Stress in Farm Animals : An Integrative Approach. Martinus Nijhoff Publishers, Dordrecht/Boston/Lancaster.

Broom D.M., Johnson K.G., 1993. Stress and Animal Welfare. Chapman and Hall, Animal Behaviour Series, London, $211 \mathrm{pp}$.

Cronin G.M., Wiepkema R., Van Ree J.M., 1985. Endogenous opioids are involved in abnormal stereotyped behaviours of tethered sows. Neuropeptides, 6, 527-530. 
Dantzer R., Mormède P., 1978. Behavioural and pituitary-adrenal characteristics of pigs differing by their susceptibility to the malignant hyperthermia syndrome induced by halothane anesthesia. 1.Behavioural measures. Ann. Rech. Vét., 9, 559-567.

Dantzer R., Mormède P., 1979. Le stress en élevage intensif. Masson, Paris, 117 pp.

Dantzer R., Mormède P., 1983. Stress in farm animals : a need for reevaluation. J. Anim. Sci., 57, 618.

Dantzer R., Mormède P., Bluthe R.M., Soissons J., 1983. The effect of different housing conditions on behavioural and adrenocortical reactions in veal calves. Reprod. Nutr. Develop., 23, 501-508.

Dawkins M.S., 1983a. La Souffrance animale. Editions du Point Vétérinaire, Maisons-Alfort, 152 pp.

Dawkins M.S., 1983b. Battery hens name their price : consumer demand theory and the measurement of ethological «needs ». Anim. Behav., 31, 1195-1205

De Wilt J.G., 1985. Behaviour and Welfare of Veal Calves in Relation to Husbandry Systems. IMAG, Wageningen, $137 \mathrm{pp}$

Dellmeier G.R., Friend T.H., Gbur E.E., 1985. Comparison of four methods of calf confinement. II. Behavior. J. Anim. Sci., 60, 1102-1109.

de Passillé A.M., Rushen J., 1995. Effects of spatial restriction and behavioural deprivation on openfield responses, growth and adrenocortical reactivity of calves. Proceedings of the 29th International Congress of the International Society for Applied Ethology, 3-5 august 1995, Exeter, UK, 207.

Eddison J.C., 1995. Appropriate experimental design for the application of applied ethological research. Proceedings of the 29th International Congress of the International Society for Applied Ethology, 3-5 August 1995, Exeter, UK, 53-54.

Faure J.M., 1986. Operant determination of the cage and feeder size preferences of the laying hen. Appl. Anim. Behav. Sci., 15, 325-336.

Faure J.M., Mills A., 1995. Bien-être et comportement chez les oiseaux domestiques. INRA Prod. Anim., 8, 57-67.

Friend T.H., Dellmeier G.R., Gbur E.E., 1985. Comparison of four methods of calf confinement. I. Physiology. J. Anim. Sci., 60, 1095-1101.

Gonzalez R., 1989. Contribution à l'étude de l'effet de l'introduction de femelles dans un groupe de mâles, sur les sécrétions endocrines du mâle chez les Ovins. Thèse de Doctorat de l'Université de Paris VI, $93 \mathrm{pp}$

Haltmeyer G.C., Denenberg V.H., Zarrow M.X., 1967. Modification of the plasma corticosterone response as a function of infantile stimulation and electric shock parameters. Physiology and Behavior, 2, 61-63.

Hammel K.L., Metz J.H.M., Mekking P., 1988. Sucking behaviour of dairy calves fed milk ad libitum by bucket or teat. Appl. Anim. Behav. Sci., 20, 275285.

Hughes B.O., Black A.J., 1973. The preference of domestic hens for different types of battery cage floor. Br. Poult. Sci., 14, 615-619.
Jensen P., 1995. Stress as a motivational state. Acta Agric. Scand., à paraître.

Kant G.J., Eggleston T., Landman-Roberts L., Kenion C.C., Driver G.C., Meyerh, 1985. Habituation to repeated stress is stressor specific. Pharmacology Biochemistry and Behavior, 22, 631-634.

Kennes D., Odberg F.O., Bouquet Y., De Rycke P.H., 1988. Changes in naloxone and haloperidol effects during the development of captivity-induced jumping stereotypy in bank voles. Eur. J. Pharmocol., $153,19-24$

Ketelaar-de Lauwere C.C., Smits A.C., 1991. Spatial requirements of individually housed veal calves of 175 to $300 \mathrm{~kg}$. In : J.H.M. Metz and C.M. Grœnestein (Editors), New Trends in Veal Calf Production, 49-53. EAAP Publication, 52, Pudoc, Wageningen.

Kooijman J., Wierenga H.K., Wiepkema P.R., 1991. Development of abnormal oral behaviour in grouphoused veal calves : effects of roughage supply. In : J.H.M. Metz and C.M. Gronestein (Editors), New Trends in Veal Calf Production, 54-58. EEAP Publication 52, Pudoc, Wageningen.

Kopp M.B., Friend T.H., Dellmeier G.R., 1986. Effect of feeding method on non nutritive oral activities in Holstein calves. J. Dairy Sci., 69, 3094-3099.

Korte S.M., Bouws G.A.H., Koolhaas J.M., Bohus B., 1992. Neurœndocrine and behavioral responses during conditioned active and passive behavior in the defensive burying/probe avoidance paradigm : effect of ipsapirone. Physiol. Behav., 52, 355-361.

Le Neindre P., 1993. Evaluating Housing Systems for Veal Calves. J. Anim. Sci., 71, 1345-1354.

Mason G.J., 1993. Forms of stereotypic behaviour. In : A.B. Lawrence and J. Rushen (Editors), Stereotypic Animal Behaviour. Fundamentals and Applications to Welfare, 7-40. Cab International, Oxon/ Tucson/Kuala Lumpur/Curepe.

Matthews L.R., Temple W., 1979. Concurrent schedule assessment of food preference in cows. J. Exp. Anal. Behav., 32, 245-254.

Mormède P., Dantzer R., 1977. Effects of dexamethasone on fear conditioning in pigs. Behav. Biol., $21,225-235$.

Odberg F.O., 1987. The influence of cage size and environmental enrichment on the development of stereotypies in bank voles (Clethrionomys glareolus). Behav Processes, 14, 155-173.

Rushen J., 1991. Problems associated with the interpretation of physiological data in the assessment of animal welfare. Appl. Anim. Behav., 28, 381.

Rutter S.M., 1995. To what extent animal welfare can be measured ? Proceedings of the 29th International Congress of the International Society for Applied Ethology, 3-5 August 1995, Exeter, UK, 5758

Sahakian B.J., Robbins T.W., Morgan M.J., Iversen S.D., 1975. The effects of psychomotor stimulants on stereotypy and locomotor activity in socially-deprived and control rats. Brain Res., 84, 195-205.

Savory J.C., Seawright E., Watson A., 1992. Stereotyped behaviour in broiler breeders in relation to husbandry and opioid receptor blockade. Appl. Anim. Behav. Sci., 32, 349-360. 
Smits A.C., Ham P.J.M., 1988. (Group housing of veal calves, a study on commercial farms) Praktijkonderzœk grœpshuisvesting vleeskalveren. IMAG Rapport $\mathrm{n}^{\circ} 105$, Wageningen, $8 \mathrm{pp}$.

Stephens D.B., 1980. Stress and its measurement in domestic animals : a review of behavioral and physiological studies under field and laboratory situations. Adv. Vet. Sci., Comp. Med., 24, 179-210.

Terlouw E.M.C., Lawrence A.B., Illius A.W., 1991. Influences of feeding level and physical restriction on development of stereotypies in sows. Anim. Behav., 42, 981-991.

Van de Braak A.E., Kleinhout G.R., 1991. Logement des veaux de boucherie en logettes. Aspects zootechniques et économiques des expériences néerlandaises. Conférence AFTAA, 18-19 juin 1991, Paris, 8 pp.

Veissier I., Le Neindre P., Trillat G., 1989. The use of circadian behaviour to measure adaptation of calves to changes in their environment. Appl. Anim. Behav. Sci., 22, 1-12.

Veissier I., Le Neindre P., Gesmier V., 1994a. Welfare and group housing of veal calves. $45^{\mathrm{e}}$ Réunion annuelle de la Fédération Européenne de Zootechnie, Edimbourg, 5-8 Septembre 1994, 10 pp.

Veissier I., Gesmier V., Le Neindre P., Gautier J.Y., Bertrand G., 1994 (b). The effects of rearing in individual crates on subsequent social behaviour of veal calves. Appl. Anim. Behav. Sci., 41, 199-210.

Webster A.J.F., 1991. Control of infectious disease in housed veal calves. In : J.H.M. Metz and C.M. Grœnestein (Editors), New Trends in Veal Calf Production, 103-112. EAAP Publication, 52, Pudoc, Wageningen.

Webster A.J.F., Saville C., Church B.M., Gnanasakthy A., Moss R., 1985. The effect of different rearing systems on the development of calf behaviour. Br. Vet. J., 141, 249-264.
Behavioural analyses in the studies on veal calves welfare.

The welfare of an animal is fulfilled when it can adapt to the environment. This will be the case if the environment is ideal so that all the needs are satisfied or if the environment is not ideal but that the animal can find a way to adapt whithout suffering or exhaustion. Anthropomorphism ought to be avoided in evaluating animal welfare. The role of the scientist is to determine objective measurements of adaptation. These consist of indices of health status, production parameters, neuro-endocrine responses to stress and behavioural deviations. Behavioural indices are very helpful due to their high sensitivity and specificity. In addition, the magnitude of behavioural alteration often varies with the severity of the constraint.

Behavioural indices can be used as predictors (the circadian rhythm of activity is modified before clinical symptoms of a disease) and to detect mild variations between life conditions (the reduction of social contacts enhances behavioural reactivity without physiological change). Behavioural indices can give information on the nature of constraints. For example, lying postures of calves can be used to assess the lack of space and oral non-nutritive activities can be used to assess feeding systems. In some cases, there is a close relation between the frequency of some activities or postures and the severity of the constraint. This relation is more easily highlighted when synthetic behavioural scores are calculated. However, to choose between several rearing conditions, one should rely not only on behaviour but on a range of indices.

These aspects are illustrated with examples on calves, the most striking constraints upon veal calves being restriction of place, absence of solid food and social isolation.

VEISSIER I., 1996. Intérêts de l'analyse comportementale dans les études de bien-être : le cas des veaux de boucherie. INRA Prod. Anim., 9 (2), 103111. 